\title{
Diagnostic Laparoscopy with Ultrasound Still Has a Role in the Staging of Pancreatic Cancer: A Systematic Review of the Literature
}

\author{
Jordan Levy, ${ }^{1}$ Mehdi Tahiri, ${ }^{1,2}$ Tsafrir Vanounou, ${ }^{1,2}$ Geva Maimon, ${ }^{2}$ and Simon Bergman ${ }^{1,2}$ \\ ${ }^{1}$ Division of General Surgery, Jewish General Hospital, McGill University, Montreal, QC, Canada H3T 1E2 \\ ${ }^{2}$ Lady Davis Institute for Medical Research, Montreal, QC, Canada H3T 1E2 \\ Correspondence should be addressed to Simon Bergman; simon.bergman@mcgill.ca
}

Received 3 December 2015; Revised 25 February 2016; Accepted 8 March 2016

Academic Editor: Attila Olah

Copyright ( $\odot 2016$ Jordan Levy et al. This is an open access article distributed under the Creative Commons Attribution License, which permits unrestricted use, distribution, and reproduction in any medium, provided the original work is properly cited.

Background. The reported incidence of noncurative laparotomies for pancreatic cancer using standard imaging (SI) techniques for staging remains high. The objectives of this study are to determine the diagnostic accuracy of diagnostic laparoscopy with ultrasound (DLUS) in assessing resectability of pancreatic tumors. Study Design. We systematically searched the literature for prospective studies investigating the accuracy of DLUS in determining resectability of pancreatic tumors. Results. 104 studies were initially identified and 19 prospective studies (1,573 patients) were included. DLUS correctly predicted resectability in $79 \%$ compared to $55 \%$ for SI. DLUS prevented noncurative laparotomies in 33\%. Of those, the most frequent DLUS findings precluding resection were liver metastases, vascular involvement, and peritoneal metastases. DLUS had a morbidity rate of $0.8 \%$ with no mortalities. DLUS remained superior to SI when analyzing studies published only in the last five years (100\% versus $81 \%)$, enrolling patients after the year 2000 (74\% versus 58\%), or comparing DLUS to modern multidimensional CT (100\% versus 78\%). Conclusion. DLUS seems to still have a role in the preoperative staging of pancreatic cancer. With its ability to detect liver metastases, vascular involvement, and peritoneal metastases, the use of DLUS leads to less noncurative laparotomies.

\section{Introduction}

Pancreatic cancer represents an aggressive disease that is resectable in only $10-20 \%$ of patients at the time of diagnosis $[1,2]$. While resection can be curative in some, it may also be abandoned intraoperatively due to the presence of occult advanced disease [3]. Careful selection of patients for surgery is important in order to avoid unnecessary procedures and their associated morbidities. In addition, with the advent of minimally invasive procedures for symptomatic relief and palliation, such as endoscopic and percutaneous biliary stenting and laparoscopic duodenal and biliary bypass, the need to correctly identify unresectable patients prior to laparotomy has been further emphasized [4].

Diagnostic laparoscopy (DL) was introduced in many preoperative staging algorithms for pancreatic carcinoma over 20 years ago [5]. Its value seemed to have been considerably enhanced with the adjunct of laparoscopic ultrasound
(LUS) $[4,6]$. Despite the growing body of research in the use of diagnostic laparoscopy with ultrasound (DLUS) for preoperative staging of pancreatic cancers, its application remains controversial [7]. Several studies support its use, as it is a sensitive tool in detecting small hepatic lesions, vascular invasion, and malignant lymphadenopathy [8]. However, many have argued, especially with the advent of multidimensional computed tomography (CT), that standard imaging (SI) modalities may be sufficient and just as reliable in staging of pancreatic cancer, obviating the need of an additional operative procedure [9].

We performed a systematic review of prospective studies investigating the use of DLUS in staging pancreatic cancer. The objectives of this study are (1) to determine the diagnostic accuracy of DLUS in assessing resectability of pancreatic tumors, (2) to compare the reported resection rates of DLUS to standard preoperative imaging, and (3) to determine how the accuracy of these modalities has evolved over time. 


\section{Methods}

2.1. Data Sources and Searches. A focused literature search using Medline and EMBASE databases, through June 2014, was conducted. Prospective studies evaluating the accuracy of diagnostic laparoscopy followed by laparoscopic ultrasound in determining resectability of pancreatic cancer were included. The search strategy combined the terms "laparoscopic ultraso" " and "pancrea*" and "cancer" or "tumor" or "malignancy" and "stage" or "staging" in the English language. This strategy was complemented by manually searching the references of the studies identified in the primary search. Study eligibility criteria were (1) that it was prospective; (2) that its objective was to investigate the accuracy of DLUS in determining resectability of pancreatic tumors; (3) that it reported intraoperative DLUS findings; and (4) that surgery was considered the gold standard for resectability.

2.2. Data Extraction. Data from each study was independently extracted by two reviewers. Disagreements were resolved by consensus or, when necessary, by a third reviewer. The reviewers systematically extracted information on author, date of publication, institution, study design, enrolment years, patient demographics, type of preoperative imaging, laparoscopic ultrasound probe and monitor specifications, morbidity associated with DLUS, and failure rates in performing DLUS. The reviewers also extracted statistical data, including sensitivity, specificity, and predictive values of DLUS and SI. We respected the following rigorous criteria for our analysis: (1) all patients declining or unfit (determined by the surgical team at that time) for DLUS or laparotomy were excluded. (2) All patients in whom laparoscopic ultrasound was not achieved were excluded, unless diagnostic laparoscopy had already proven unresectability before LUS was required. (3) In certain studies, patients were classified as "doubtfully resectable"; those patients were treated similarly to the resectable group and were thus included in our study as such. (4) Benign lesions discovered at DLUS or laparotomy were considered as "resected" for the purpose of the analysis.

2.3. Statistical Analysis. Both imaging techniques, DLUS and SI, are being used to determine the resectability of a pancreatic cancer. Hence, for our purposes, the term "true positive" refers to a cancer that was deemed resectable by a staging technique and was actually resected. Similarly, a "true negative" refers to a cancer deemed unresectable by SI or DLUS and confirmed as unresectable according to operative findings, cytopathology, frozen section, or grossly suspicious findings during either staging technique. Sensitivity is defined as the number of true positives over the number of resectable cancers. Specificity is defined as the number of true negatives over the total number of unresectable cancers. The positive predictive value is the number of true positives over the total number of cancers deemed resectable by imaging. Negative predictive value is the number of true negatives over the total number of cancers deemed unresectable by imaging. Our measure of resection rate is equivalent to the positive predictive value, as defined above. To calculate the overall resection rate across all applicable studies, the data were weighted according to each study's sample size.

\section{Results}

3.1. Study Selection and Baseline Characteristics. Study selection occurred according to the Preferred Reporting Items for Systematic Review and Meta-Analyses (PRISMA) diagram (Figure 1). The search initially identified a total of 99 abstracts, with additional five abstracts found after a manual search through the references. These abstracts were reviewed and screened for relevance. 43 full-text and potentially relevant articles were retrieved and evaluated for eligibility following exclusion of review articles $(n=29)$, nonrelevant articles $(n=24)$, conference outlines or abstracts $(n=4)$, letters to the editor $(n=2)$, critical appraisal $(n=1)$, and duplicate abstract $(n=1)$. Of the 43 full-text studies retrieved, 18 studies met the inclusion criteria and were included in the analysis. Studies were excluded because they did not provide relevant analytical data necessary for the calculation of the sensitivity and specificity of DLUS as a diagnostic tool $(n=20)$ or were not prospective studies $(n=5)$. One of the 18 prospective studies included in the systematic review reported a two-part study occurring at different times on different study populations [10]. It was thus considered as two separate studies, bringing the total to 19 prospective studies and 1,573 patients.

Eleven of 19 studies were published after January 1, 2000. The average patient age ranged from 55 to 66 years old. The percentage of male patients ranged from 25 to $64 \%$. The location of the pancreatic tumor was found most commonly in the pancreatic head, followed by the ampullary region, body, and tail, and rarely in the uncinate process (Table 1).

3.2. Execution of Preoperative Staging. CT scan was the investigation of choice in the assessment of resectability in all but one study (18/19), which was completed in a center where mesenteric angiography was frequently performed [25]. 79\% (15/19) of studies reported using at least one additional staging procedure following CT $[4,6,11,13-20,22-$ 25]: abdominal ultrasound (15/19) [4, 6, 11, 13-20, 22-25], endoscopic retrograde pancreatography $(10 / 19)[4,6,14,16-$ 20, 22, 24], endoscopic ultrasound (5/19) [13, 14, 18, 19, 23], visceral angiography (7/19) $[4,6,14,16,23-25]$, and magnetic resonance imaging (MRI) (5/19) [14-17, 20], although the additional procedures were not performed in all patients.

Diagnostic laparoscopy was first carried out to explore the peritoneal cavity in search of malignant ascites, peritoneal metastases, visceral implants, or suspicious lymph nodes. The LUS probe was then inserted. Most often, the probe used linear array with a frequency of $5-7.5 \mathrm{MHz}$ and frequently had Doppler capabilities. The liver was scanned in search of undiagnosed micrometastases and the biliary tree explored for any abnormalities. The pancreas was scanned to better characterize the primary lesion and determine local extensions into peripancreatic tissues including duodenum, mesocolon, stomach, and spleen. In less than one-third of the studies did the authors explicitly report exploring 


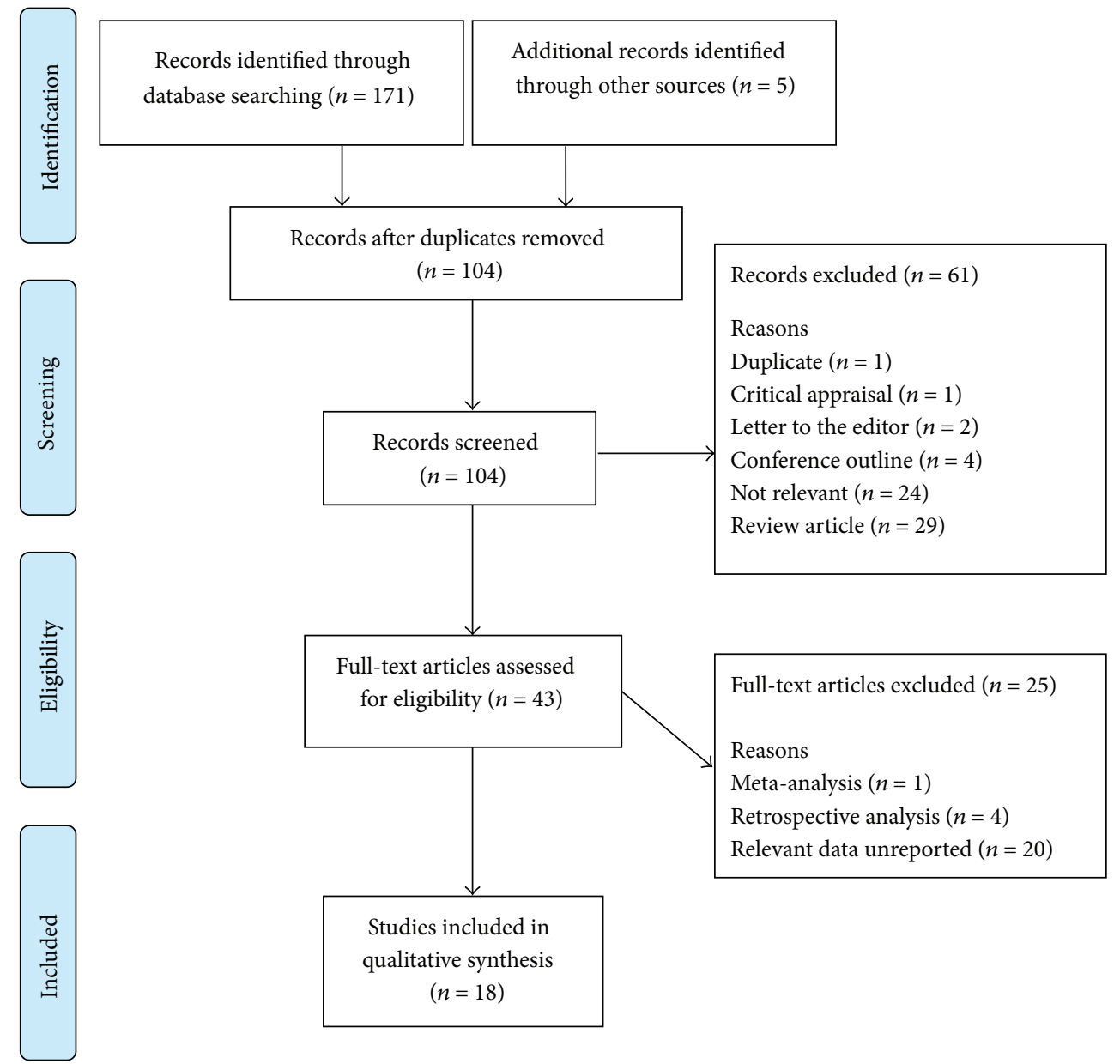

FIGURE 1: Search diagram.

the lesser sac by retroduodenal or infragastric approaches. Blood vessels, including the celiac axis, superior mesenteric artery, and the portal venous system, were characterized according to their relation to the tumor and whether they were encased, thrombosed, stenosed, infiltrated, or frankly invaded. Associated lymph node basins were also investigated.

Nine studies described DLUS timing $[6,11-13,16,18,19$, $21,24]$. In five studies it occurred as a separate procedure prior to laparotomy $[6,12,18,19,24]$; in two studies it occurred in the same setting immediately prior to laparotomy $[11,16]$. In two studies it occurred both immediately before and as a separate procedure $[13,21]$. Procedure time varied between 15 and 90 minutes depending on surgeon experience and whether biopsies and lesser sac dissection were performed.

3.3. Morbidity and Mortality. Complication rates were minimal at $0.8 \%$ (9/1076), including 2 port-site hemorrhages, 2 episodes of pancreatitis, 2 wound infections, 1 enterotomy, 1 aspiration pneumonia, and 1 bile leak following biopsy $[4,6,16,17,24]$. There were no procedure-related mortalities.

3.4. Resectability Criteria. Nonresectability criteria differed between studies. All studies considered liver and peritoneal and other distant metastases unresectable. Seven studies only considered distant lymphadenopathy as unresectable $[11,13,14,16,17,21,22]$ while two studies included regional involvement $[4,19]$; the rest of the studies did not specify. Size was only considered in three studies $[4,12,25]$. Most studies considered any vascular involvement as unresectable, except four studies in which some degree of portal vein or superior mesenteric vein was considered resectable [14, 16, 22, 24]. All but one study [11] discussed confirmation of nonresectability due to liver, peritoneal, or lymph node metastases by biopsy proven histopathology.

3.5. Rates of Resection. Studies including data on SI are summarized in Table 2. CT was used in 99.7\% (1569/1573) of patients to determine resectability; 4 patients underwent angiography without CT. Of these, the data for 1442 patients from 15 studies were available for analysis $[4,6,10-14,16$, $18,19,21,22,24,25]$. Eight of 15 studies only included "SI resectable" patients in their analysis without presenting the initial study population screened by SI, precluding a sensitivity and specificity analysis $[4,10,11,18,21,24,25]$. Following imaging, 911 patients were considered resectable and of these, only 505 were resected at laparotomy, corresponding to a 
TABLE 1: Study characteristics.

\begin{tabular}{|c|c|c|c|c|c|c|c|c|}
\hline Author & $\begin{array}{c}\text { Year of } \\
\text { publication }\end{array}$ & $\begin{array}{c}\text { Years of } \\
\text { enrolment }\end{array}$ & $\begin{array}{l}\text { Study } \\
\text { design }\end{array}$ & Country & Sample size & Mean age & $\%$ male & Location/tumor type \\
\hline $\begin{array}{l}\text { Barabino et } \\
\text { al. [10] }\end{array}$ & 2011 & 1995-1999 & Prospective & Italy & 40 & NR & NR & Periampullary 40 \\
\hline $\begin{array}{l}\text { Barabino et } \\
\text { al. [10] }\end{array}$ & 2011 & $2002-2007$ & Prospective & Italy & 64 & NR & NR & Periampullary 64 \\
\hline $\begin{array}{l}\text { Piccolboni et } \\
\text { al. [11] }\end{array}$ & 2010 & 2005-2008 & Prospective & Italy & 48 & NR & NR & NR \\
\hline $\begin{array}{l}\text { Doucas et al. } \\
{[12]}\end{array}$ & 2007 & 2001-2004 & Prospective & UK & 100 & 63 & $52 \%$ & Head 90 , body, or tail 10 \\
\hline $\begin{array}{l}\text { Fristrup et al. } \\
{[13]}\end{array}$ & 2006 & $2002-2004$ & Prospective & Denmark & 148 & $66^{*}$ & $54 \%$ & NR \\
\hline $\begin{array}{l}\text { Doran et al. } \\
{[14]}\end{array}$ & 2004 & 1997-2002 & Prospective & UK & 239 & $64^{*}$ & $60 \%$ & NR \\
\hline $\begin{array}{l}\text { Zhao et al. } \\
\text { [15] }\end{array}$ & 2003 & NR & Prospective & China & 22 & 55 & $64 \%$ & Head 22 \\
\hline $\begin{array}{l}\text { Kwon et al. } \\
{[16]}\end{array}$ & 2002 & $1996-2000$ & Prospective & Japan & 118 & 59 & $64 \%$ & Head 39, body 13 \\
\hline $\begin{array}{l}\text { Lavonius et } \\
\text { al. [17] }\end{array}$ & 2001 & 1997-1999 & Prospective & Finland & 27 & 63 & $48 \%$ & $\begin{array}{c}\text { Head 21, body 2, } \\
\text { chronic pancreatitis } 4\end{array}$ \\
\hline $\begin{array}{l}\text { Taylor et al. } \\
{[18]}\end{array}$ & 2001 & $1996-2000$ & Prospective & $\mathrm{UK}$ & 51 & 66 & $57 \%$ & Head 42, ampullary 9 \\
\hline $\begin{array}{l}\text { Schachter et } \\
\text { al. [19] }\end{array}$ & 2000 & 1996-1999 & Prospective & Israel & 94 & 63 & $46 \%$ & $\begin{array}{l}\text { Head 40, body, or tail } 19 \\
\text { UP 5, ampullary } 3\end{array}$ \\
\hline $\begin{array}{l}\text { Velasco et al. } \\
{[20]}\end{array}$ & 2000 & NR & Prospective & USA & 33 & NR & NR & (1) \\
\hline $\begin{array}{l}\text { Norton et al. } \\
{[21]}\end{array}$ & 1999 & NR & Prospective & USA & 50 & NR & NR & NR \\
\hline $\begin{array}{l}\text { Minnard et } \\
\text { al. [22] }\end{array}$ & 1998 & 1993-1995 & Prospective & USA & 90 & $65^{*}$ & $47 \%$ & $\begin{array}{l}\text { Head } 64 \text {, body } 19 \text {, } \\
\text { ampullary } 4 \text {, tail } 3\end{array}$ \\
\hline $\begin{array}{l}\text { Champault et } \\
\text { al. [23] }\end{array}$ & 1997 & 1994-1996 & Prospective & France & 26 & 61 & $46 \%$ & Head 26 \\
\hline $\begin{array}{l}\text { Pietrabissa et } \\
\text { al. [24] }\end{array}$ & 1996 & 1994-1995 & Prospective & Italy & 21 & 65 & $62 \%$ & Head 14, body, or tail 7 \\
\hline $\begin{array}{l}\text { Bemelman et } \\
\text { al. [6] }\end{array}$ & 1995 & 1993-1994 & Prospective & Netherlands & 350 & NR & NR & Head 60, ampullary 13 \\
\hline John et al. [4] & 1995 & 1991-1993 & Prospective & UK & 40 & $59^{*}$ & $45 \%$ & NR \\
\hline $\begin{array}{l}\text { Murugiah et } \\
\text { al. [25] }\end{array}$ & 1993 & 1991-1992 & Prospective & $\mathrm{UK}$ & 12 & 58 & $25 \%$ & Head 12 \\
\hline
\end{tabular}

* Median age.

AdenoCA = adenocarcinoma, NOS = not otherwise specified, CCA $=$ cholangiocarcinoma, NET $=$ neuroendocrine tumor, $\mathrm{UP}=$ uncinate process, NR $=$ not reported.

resection rate of $55 \%(29 \%-85 \%)[4,6,10-14,16,18,19,21$, 22, 24, 25].

Table 3 summarizes DLUS data. 1076 patients were initially considered for DLUS. However, five patients declined further investigations and were excluded from the study; failures due to dense adhesions occurred in nine patients, while 12 patients were deemed unfit for surgery and were also excluded from formal analysis. Ultimately, 1050 patients were investigated using DLUS. 646 patients were deemed resectable and 513 were finally resected, corresponding to a resection rate of $79 \%(41 \%-100 \%)$. Of note, even those studies employing additional diagnostic procedures following CT did not show superior accuracy than DLUS. Such complementary studies, such as EUS, once represented an important role in pancreatic cancer staging and have now fallen out of favor with certain institutions recommending against its routine use in staging [26].

3.6. DLUS versus SI. 14 studies presented data on SI and DLUS findings in a sequential manner such that the study population could be followed up from SI to DLUS [4, 6, 10-14, $16,18,19,21,24,25]$. In 781 patients deemed resectable by SI, DLUS correctly prevented noncurative laparotomies in 254 (33\%). In this group, the most common findings precluding resection were liver metastases, vascular involvement, and peritoneal metastases. 
TABLE 2: Analysis of SI.

\begin{tabular}{|c|c|c|c|c|c|c|c|}
\hline \multirow{2}{*}{ Author } & \multirow{2}{*}{ Year } & \multirow{2}{*}{ \# receiving SI } & \multirow{2}{*}{ Analysis sample } & \multicolumn{4}{|c|}{ Resectability } \\
\hline & & & & Sensitivity & Specificity & PPV & NPV \\
\hline Barabino et al. [10] & 2011 & $40^{*}$ & 40 & NA & NA & $33 \%(13 / 40)$ & NA \\
\hline Barabino et al. [10] & 2011 & $64^{*}$ & 64 & NA & NA & $78 \%(50 / 64)$ & NA \\
\hline Piccolboni et al. [11] & 2010 & $48^{*}$ & 48 & NA & NA & $85 \%(41 / 48)$ & NA \\
\hline Doucas et al. [12] & 2007 & 100 & 94 & $71 \%(20 / 28)$ & $26 \%(17 / 66)$ & $29 \%(20 / 69)$ & $68 \%(17 / 25)$ \\
\hline Fristrup et al. [13] & 2006 & 148 & 148 & $100 \%(38 / 38)$ & $64 \%(70 / 110)$ & $49 \%(38 / 78)$ & $100 \%(70 / 70)$ \\
\hline Doran et al. [14] & 2004 & 239 & 227 & $96 \%(127 / 132)$ & $46 \%(44 / 95)$ & $71 \%(127 / 178)$ & $90 \%(44 / 49)$ \\
\hline Zhao et al. [15] & 2003 & 22 & NR & NA & NA & NA & NA \\
\hline Kwon et al. [16] & 2002 & 118 & 118 & $100 \%(39 / 39)$ & $84 \%(66 / 79)$ & $75 \%(39 / 52)$ & $100 \%(66 / 66)$ \\
\hline Lavonius et al. [17] & 2001 & 27 & NR & NA & NA & NA & NA \\
\hline Taylor et al. [18] & 2001 & $51^{*}$ & 49 & NA & NA & $53 \%(26 / 49)$ & NA \\
\hline Schachter et al. [19] & 2000 & 94 & 94 & $100 \%(33 / 33)$ & $44 \%(27 / 61)$ & $49 \%(33 / 67)$ & $100 \%(27 / 27)$ \\
\hline Velasco et al. [20] & 2000 & 33 & NR & NA & NA & NA & NA \\
\hline Norton et al. [21] & 1999 & $50^{*}$ & 50 & NA & NA & $52 \%(26 / 50)$ & NA \\
\hline Minnard et al. [22] & 1998 & 90 & 90 & $100 \%(40 / 40)$ & $34 \%(17 / 50)$ & $55 \%(40 / 73)$ & $100 \%(17 / 17)$ \\
\hline Champault et al. [23] & 1997 & 26 & NR & NA & NA & NA & NA \\
\hline Pietrabissa et al. [24] & 1996 & $21^{*}$ & 21 & NA & NA & $62 \%(13 / 21)$ & NA \\
\hline Bemelman et al. [6] & 1995 & 350 & 347 & $100 \%(22 / 22)$ & $85 \%(277 / 325)$ & $31 \%(22 / 70)$ & $100 \%(277 / 277)$ \\
\hline John et al. [4] & 1995 & $40^{*}$ & 40 & NA & NA & $30 \%(12 / 40)$ & NA \\
\hline Murugiah et al. [25] & 1993 & $12^{*}$ & 12 & NA & NA & $42 \%(5 / 12)$ & NA \\
\hline
\end{tabular}

${ }^{*}$ Size of initial population screened not available. Only patients deemed resectable as per SI were included.

NA = Not applicable, NR = Not Reported.

TABLe 3: Analysis of DLUS.

\begin{tabular}{|c|c|c|c|c|c|c|}
\hline \multirow{2}{*}{ Author } & \multirow{2}{*}{ Year } & \multirow{2}{*}{ Analysis sample } & \multicolumn{4}{|c|}{ Resectability } \\
\hline & & & Sensitivity & Specificity & PPV (resection rate) & NPV \\
\hline Barabino et al. [10] & 2011 & 40 & $100 \%(13 / 13)$ & $93 \%(25 / 27)$ & $87 \%(13 / 15)$ & $100 \%(25 / 25)$ \\
\hline Barabino et al. [10] & 2011 & 9 & $100 \%(1 / 1)$ & $100 \%(8 / 8)$ & $100 \%(1 / 1)$ & $100 \%(8 / 8)$ \\
\hline Piccolboni et al. [11] & 2010 & 48 & $100 \%(41 / 41)$ & $100 \%(7 / 7)$ & $100 \%(41 / 41)$ & $100 \%(7 / 7)$ \\
\hline Doucas et al. [12] & 2007 & 94 & $100 \%(28 / 28)$ & $64 \%(42 / 66)$ & $54 \%(28 / 52)$ & $100 \%(42 / 42)$ \\
\hline Fristrup et al. [13] & 2006 & 78 & $100 \%(38 / 38)$ & $65 \%(26 / 40)$ & $73 \%(38 / 52)$ & $100 \%(26 / 26)$ \\
\hline Doran et al. [14] & 2004 & 227 & $98 \%(130 / 132)$ & $57 \%(54 / 95)$ & $76 \%(130 / 171)$ & $96 \%(54 / 56)$ \\
\hline Zhao et al. [15] & 2003 & 22 & $100 \%(9 / 9)$ & $92 \%(12 / 13)$ & $90 \%(9 / 10)$ & $100 \%(12 / 12)$ \\
\hline Kwon et al. [16] & 2002 & 52 & $100 \%(39 / 39)$ & $100 \%(13 / 13)$ & $100 \%(39 / 39)$ & $100 \%(13 / 13)$ \\
\hline Lavonius et al. [17] & 2001 & 24 & $100 \%(11 / 11)$ & $69 \%(9 / 13)$ & $73 \%(11 / 15)$ & $100 \%(9 / 9)$ \\
\hline Taylor et al. [18] & 2001 & 49 & $100 \%(26 / 26)$ & $91 \%(21 / 23)$ & $93 \%(26 / 28)$ & $100 \%(21 / 21)$ \\
\hline Schachter et al. [19] & 2000 & 67 & $100 \%(33 / 33)$ & $88 \%(30 / 34)$ & $89 \%(33 / 37)$ & $100 \%(30 / 30)$ \\
\hline Velasco et al. [20] & 2000 & 33 & $100 \%(22 / 22)$ & $82 \%(9 / 11)$ & $92 \%(22 / 24)$ & $100 \%(9 / 9)$ \\
\hline Norton et al. [21] & 1999 & 50 & $100 \%(26 / 26)$ & $92 \%(22 / 24)$ & $93 \%(26 / 28)$ & $100 \%(22 / 22)$ \\
\hline Minnard et al. [22] & 1998 & 90 & $100 \%(40 / 40)$ & $98 \%(49 / 50)$ & $98 \%(40 / 41)$ & $100 \%(49 / 49)$ \\
\hline Champault et al. [23] & 1997 & 26 & $100 \%(5 / 5)$ & $100 \%(21 / 21)$ & $100 \%(5 / 5)$ & $100 \%(21 / 21)$ \\
\hline Pietrabissa et al. [24] & 1996 & 21 & $100 \%(13 / 13)$ & $100 \%(8 / 8)$ & $100 \%(13 / 13)$ & $100 \%(8 / 8)$ \\
\hline Bemelman et al. [6] & 1995 & 70 & $100 \%(22 / 22)$ & $33 \%(16 / 48)$ & $41 \%(22 / 54)$ & $100 \%(16 / 16)$ \\
\hline John et al. [4] & 1995 & 38 & $92 \%(11 / 12)$ & $88 \%(23 / 26)$ & $79 \%(11 / 14)$ & $96 \%(23 / 24)$ \\
\hline Murugiah et al. [25] & 1993 & 12 & $100 \%(5 / 5)$ & $86 \%(6 / 7)$ & $83 \%(5 / 6)$ & $100 \%(6 / 6)$ \\
\hline
\end{tabular}

3.7. DLUS versus DL. The added benefit of laparoscopic ultrasound (LUS) to diagnostic laparoscopy (DL) was investigated and clearly reported in three studies. In these studies, diagnostic laparoscopy with ultrasound (DLUS) identified 64 unresectable patients, of which 37 were discovered using ultrasound after being overlooked by diagnostic laparoscopy (DL) alone. Signifying that $58 \%$ of these accurate staging procedures were directly attributable to the addition of ultrasound to diagnostic laparoscopy. The findings precluding resection in these 37 patients were 17 vascular involvements, 
14 liver metastases, 5 malignant lymphadenopathies, and 1 transverse mesocolon invasion $[6,13,21]$.

3.8. Controlling for Advances in Diagnostic Imaging. As imaging studies have improved substantially in recent years, subgroup analyses of studies published in the last five years, enrolling patients after 2000 and those using multidimensional CT (MDCT), were carried out. In studies published between 2009 and 2014 (two studies), the resection rates using DLUS and SI were $100 \%$ and $81 \%$ (78\%-85\%), respectively $[10,11]$. In those studies enrolling patients only after the year 2000 (four studies), the resection rates were $74 \%$ (54\%-100\%) and $58 \%(29 \%-85 \%)$ for DLUS and SI, respectively [10-13]. In the only prospective study specifically comparing DLUS to multidimensional CT (and no previous model of CT), the resection rates were $100 \%$ and $78 \%$, respectively [10].

\section{Discussion}

Currently, DLUS is not routinely used in preoperative staging of pancreatic tumors. Some institutions selectively incorporate it into staging protocols, while others do not use it at all. Our study was designed to determine the accuracy of DLUS in determining resectability of pancreatic tumors. We included only the most rigorous prospective studies, in which DLUS, SI, and laparotomy findings were clearly reported.

Overall, by weighted analysis, DLUS improved the resection rate of pancreatic malignancies from $55 \%$ to $79 \%$ with no increase in mortality and a $0.8 \%$ complication rate. DLUS remained more accurate when restricting our analysis to more recent studies, in which SI had presumably improved.

A meta-analysis published in 2010 evaluating the role of DL and LUS in the preoperative staging of pancreaticobiliary cancer demonstrated that it improved resection rates of pancreatic malignancies from $61 \%$ to $80 \%$ [27]. These results are largely consistent with our systematic review. Our study differs in that we included only prospective studies and focused on comparing operative findings and resection rates following DLUS to SI. In addition, we have updated the literature search with all eligible studies published after the meta-analysis.

4.1. Modernized Standard Imagine. It is possible that the studies included in this systematic review are not representative of modern staging techniques, as they did not all employ MDCT. It is important to acknowledge that modern techniques for CT imaging offer higher-resolution images with more detail of vascular involvement and metastatic disease. Advances in CT imaging, namely, multiphase imaging technique including noncontrast, arterial, pancreatic parenchymal, and portal venous phases with cuts less than $3 \mathrm{~mm}$ through the abdomen, have improved its ability to predict resectability of pancreatic tumors $[28,29]$. A prospective study comparing MDCT Angiography with MDCT 3D Reconstruction reported resection rates of $94 \%$ and $100 \%$, respectively. However, MDCT Angiography also overestimated unresectability in $32 \%$ of patients, which may be in part due to overestimating vascular invasions [30]. The authors suggest that older grading schemes like those presented by Lu et al. [31] and Loyer et al. [32], which assess circumferential contiguity, tissue planes, mass effects, and occlusions, may be improved by visualizing tumor infiltration and vascular smoothness. An assessment readily made by LUS.

A study investigating MDCT for pancreatic head tumors found that only $40 \%$ of their "CT resectable" group was resected and that this was due to MDCT underestimating vascular involvement and local invasion. A subgroup analysis of patients that were unequivocally resectable improved the resection rate to $56 \%$ [33].

The use of MRI has increased dramatically in recent years and is considered by some to be standard of care along with MDCT cross-sectional imaging [34]. Using MRI with a pancreas protocol, at a high volume center, leads to a resection rate of $73 \%$. The most common causes of intraoperative unresectability were vascular involvement and distant metastases, two findings aptly diagnosed by DLUS [29].

4.2. Timing and Cost Analysis. We believe that the optimal approach to include DLUS in the staging protocol is immediately prior to planned resection, which would minimize risks related to a second surgical procedure and general anesthesia. It may prove to be cost-effective as the patient would ultimately spend fewer days in hospital and most importantly decrease theoretical risk of progression in between procedures and delay in chemoradiation $[35,36]$. In a recent cost-efficacy analysis of diagnostic laparoscopy prior to laparotomy for pancreatic cancer, the authors found that the total cost for introducing diagnostic laparoscopy was $1,480 \$$ less per patient and provided better quality of life [37].

\section{Limitations}

This study has several limitations. The studies were heterogeneous, in their resectability criteria, use of multimodal imaging protocols, and the quality of their CT technology. In recent years there has been a paucity of literature on the subject and thus direct comparison of DLUS with more modern SI techniques is not possible. An important issue with DLUS is that the excellent results reported here may not be easily transferable to other centers where experience with this technique may be limited. The true benefit of DLUS may be difficult to achieve in all cases given the required expertise to perform and interpret this test correctly. In one study spanning three years, the average time to perform DLUS with lesser sac dissection in 67 patients was 30 minutes. The time to perform improved to 21 from 39 minutes in the last six months of the study [19].

\section{Conclusion}

Based on the highest quality studies available at this time, DLUS seems to still have a role in the preoperative staging of pancreatic cancer alongside SI techniques. With its ability to detect occult liver metastases, vascular involvement, and peritoneal metastases, the use of DLUS may lead to less 
noncurative laparotomies. Further research is warranted to compare DLUS to Pancreas Protocol MDCT and MRI.

\section{Abbreviations}

CT: Computed tomography

DL: Diagnostic laparoscopy

DLUS: Diagnostic laparoscopy with ultrasound

LUS: Laparoscopic ultrasound

MDCT: Multidimensional computed tomography

SI: $\quad$ Standard imaging.

\section{Disclosure}

This study was presented at SAGES 2015 and CSF 2015.

\section{Competing Interests}

The authors declare that they have no competing interests.

\section{References}

[1] A. Jemal, R. Siegel, J. Xu, and E. Ward, "Cancer statistics, 2010," CA: Cancer Journal for Clinicians, vol. 60, no. 5, pp. 277-300, 2010.

[2] J. P. Neoptolemos, D. D. Stocken, H. Friess et al., "A randomized trial of chemoradiotherapy and chemotherapy after resection of pancreatic cancer," The New England Journal of Medicine, vol. 350, no. 12, pp. 1200-1210, 2004.

[3] M. Sugiyama, H. Hagi, and Y. Atomi, "Reappraisal of intraoperative ultrasonography for pancreatobiliary carcinomas: assessment of malignant portal venous invasion," Surgery, vol. 125 , no. 2, pp. $160-165,1999$.

[4] T. G. John, J. D. Greig, D. C. Carter, and O. J. Garden, "Carcinoma of the pancreatic head and periampullary region: tumor staging with laparoscopy and laparoscopic ultrasonography," Annals of Surgery, vol. 221, no. 2, pp. 156-164, 1995.

[5] A. Cuschieri, "Laparoscopy for pancreatic cancer: does it benefit the patient," European Journal of Surgical Oncology, vol. 14, no. 1, pp. 41-44, 1988.

[6] W. A. Bemelman, L. T. de Wit, O. M. van Delden et al., "Diagnostic laparoscopy combined with laparoscopic ultrasonography in staging of cancer of the pancreatic head region," British Journal of Surgery, vol. 82, no. 6, pp. 820-824, 1995.

[7] W. Richardson, D. Stefanidis, S. Mittal, and R. D. Fanelli, "SAGES guidelines for the use of laparoscopic ultrasound," Surgical Endoscopy, vol. 24, no. 4, pp. 745-756, 2010.

[8] A. Foroutani, A. M. Garland, E. Berber et al., "Laparoscopic ultrasound vs triphasic computed tomography for detecting liver tumors," Archives of Surgery, vol. 135, no. 8, pp. 933-938, 2000.

[9] S. C. Mayo, D. F. Austin, B. C. Sheppard, M. Mori, D. K. Shipley, and K. G. Billingsley, "Evolving preoperative evaluation of patients with pancreatic cancer: does laparoscopy have a role in the current era?" Journal of the American College of Surgeons, vol. 208, no. 1, pp. 87-95, 2009.

[10] M. Barabino, R. Santambrogio, A. Pisani Ceretti, R. Scalzone, M. Montorsi, and E. Opocher, "Is there still a role for laparoscopy combined with laparoscopic ultrasonography in the staging of pancreatic cancer?" Surgical Endoscopy, vol. 25, no. 1, pp. 160-165, 2011.
[11] D. Piccolboni, F. Ciccone, A. Settembre, and F. Corcione, "Laparoscopic intra-operative ultrasound in liver and pancreas resection: analysis of 93 cases," Journal of Ultrasound, vol. 13, no. 1, pp. 3-8, 2010.

[12] H. Doucas, C. D. Sutton, A. Zimmerman, A. R. Dennison, and D. P. Berry, "Assessment of pancreatic malignancy with laparoscopy and intraoperative ultrasound," Surgical Endoscopy and Other Interventional Techniques, vol. 21, no. 7, pp. 1147-1152, 2007.

[13] C. W. Fristrup, M. B. Mortensen, T. Pless et al., "Combined endoscopic and laparoscopic ultrasound as preoperative assessment of patients with pancreatic cancer," $H P B$, vol. 8, no. 1, pp. 57-60, 2006.

[14] H. E. Doran, L. Bosonnet, S. Connor et al., "Laparoscopy and laparoscopic ultrasound in the evaluation of pancreatic and periampullary tumours," Digestive Surgery, vol. 21, no. 4, pp. 305-313, 2004.

[15] Z.-W. Zhao, J.-Y. He, G. Tan, H.-J. Wang, and K.-J. Li, "Laparoscopy and laparoscopic ultrasonography in judging the resectability of pancreatic head cancer," Hepatobiliary and Pancreatic Diseases International, vol. 2, no. 4, pp. 609-611, 2003.

[16] A. H. Kwon, H. Inui, and Y. Kamiyama, "Preoperative laparoscopic examination using surgical manipulation and ultrasonography for pancreatic lesions," Endoscopy, vol. 34, no. 6, pp. 464-468, 2002.

[17] M. I. Lavonius, S. Laine, S. Salo, P. Sonninen, and J. Ovaska, "Role of laparoscopy and laparoscopic ultrasound in staging of pancreatic tumours," Annales Chirurgiae et Gynaecologiae, vol. 90, no. 4, pp. 252-255, 2001.

[18] A. M. Taylor, S. A. Roberts, and J. McK Manson, "Experience with laparoscopic ultrasonography for defining tumour resectability in carcinoma of the pancreatic head and periampullary region," British Journal of Surgery, vol. 88, no. 8, pp. 1077-1083, 2001.

[19] P. P. Schachter, Y. Avni, M. Shimonov, G. Gvirtz, A. Rosen, and A. Czerniak, "The impact of laparoscopy and laparoscopic ultrasonography on the management of pancreatic cancer," Archives of Surgery, vol. 135, no. 11, pp. 1303-1307, 2000.

[20] J. M. Velasco, H. Rossi, T. J. Hieken, and M. Fernandez, "Laparoscopic ultrasound enhances diagnostic laparoscopy in the staging of intra-abdominal neoplasms," American Surgeon, vol. 66, no. 4, pp. 407-411, 2000.

[21] J. A. Norton, "Intraoperative methods to stage and localize pancreatic and duodenal tumors," Annals of Oncology, vol. 10, supplement 4, pp. S182-S184, 1999.

[22] E. A. Minnard, K. C. Conlon, A. Hoos, E. C. Dougherty, L. E. Hann, and M. F. Brennan, "Laparoscopic ultrasound enhances standard laparoscopy in the staging of pancreatic cancer," Annals of Surgery, vol. 228, no. 2, pp. 182-187, 1998.

[23] G. Champault, "The use of laparoscopic ultrasound in the assessment of pancreatic cancer," Wiadomości Lekarskie, vol. 50, supplement 1, part 1, pp. 195-203, 1997.

[24] A. Pietrabissa, G. Di Candio, P. C. Giulianotti, A. Carobbi, U. Boggi, and F. Mosca, "Operative technique for the laparoscopic staging of pancreatic malignancy," Minimally Invasive Therapy and Allied Technologies, vol. 5, no. 3, pp. 274-280, 1996.

[25] M. Murugiah, S. Paterson-Brown, J. A. Windsor, W. F. Anthony Miles, and O. J. Garden, "Early experience of laparoscopic ultrasonography in the management of pancreatic carcinoma," Surgical Endoscopy, vol. 7, no. 3, pp. 177-181, 1993.

[26] NCCN, Pancreatic Adenocarcinoma, Version 1, 2016, http://www .nccn.org/professionals/physician_gls/pdf/pancreatic.pdf. 
[27] D. Hariharan, V. A. Constantinides, F. E. M. Froeling, P. P. Tekkis, and H. M. Kocher, "The role of laparoscopy and laparoscopic ultrasound in the preoperative staging of pancreaticobiliary cancers-a meta-analysis," European Journal of Surgical Oncology, vol. 36, no. 10, pp. 941-948, 2010.

[28] N. J. McNulty, I. R. Francis, J. F. Platt, R. H. Cohan, M. Korobkin, and A. Gebremariam, "Multi-detector row helical CT of the pancreas: effect of contrast-enhanced multiphasic imaging on enhancement of the pancreas, peripancreatic vasculature, and pancreatic adenocarcinoma," Radiology, vol. 220, no. 1, pp. 97$102,2001$.

[29] D. M. Walters, D. J. Lapar, E. E. De Lange et al., "Pancreasprotocol imaging at a high-volume center leads to improved preoperative staging of pancreatic ductal adenocarcinoma," Annals of Surgical Oncology, vol. 18, no. 10, pp. 2764-2771, 2011.

[30] C.-H. Fang, W. Zhu, H. Wang et al., "A new approach for evaluating the resectability of pancreatic and periampullary neoplasms," Pancreatology, vol. 12, no. 4, pp. 364-371, 2012.

[31] D. S. K. Lu, H. A. Reber, R. M. Krasny, B. M. Kadell, and J. Sayre, "Local staging of pancreatic cancer: criteria for unresectability of major vessels as revealed by pancreatic-phase, thin-section helical CT,' American Journal of Roentgenology, vol. 168, no. 6, pp. 1439-1443, 1997.

[32] E. M. Loyer, C. L. David, R. A. Dubrow, D. B. Evans, and C. Charnsangavej, "Vascular involvement in pancreatic adenocarcinoma: reassessment by thin-section CT," Abdominal Imaging, vol. 21, no. 3, pp. 202-206, 1996.

[33] S. L. Smith, A. Basu, D. M. Rae, and M. Sinclair, "Preoperative staging accuracy of multidetector computed tomography in pancreatic head adenocarcinoma," Pancreas, vol. 34, no. 2, pp. 180-184, 2007.

[34] S. V. Shrikhande, S. G. Barreto, M. Goel, and S. Arya, "Multimodality imaging of pancreatic ductal adenocarcinoma: a review of the literature," $H P B$, vol. 14, no. 10, pp. 658-668, 2012.

[35] A. A. Gumbs, A. M. Rodriguez Rivera, L. Milone, and J. P. Hoffman, "Laparoscopic pancreatoduodenectomy: a review of 285 published cases," Annals of Surgical Oncology, vol. 18, no. 5, pp. 1335-1341, 2011.

[36] R. Venkat, B. H. Edil, R. D. Schulick, A. O. Lidor, M. A. Makary, and C. L. Wolfgang, "Laparoscopic distal pancreatectomy is associated with significantly less overall morbidity compared to the open technique: a systematic review and meta-analysis," Annals of Surgery, vol. 255, no. 6, pp. 1048-1059, 2012.

[37] T. T. Jayakrishnan, H. Nadeem, R. T. Groeschl et al., "Diagnostic laparoscopy should be performed before definitive resection for pancreatic cancer: a financial argument," $H P B$, vol. 17, no. 2, pp. 131-139, 2015. 


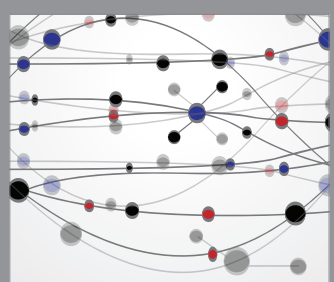

The Scientific World Journal
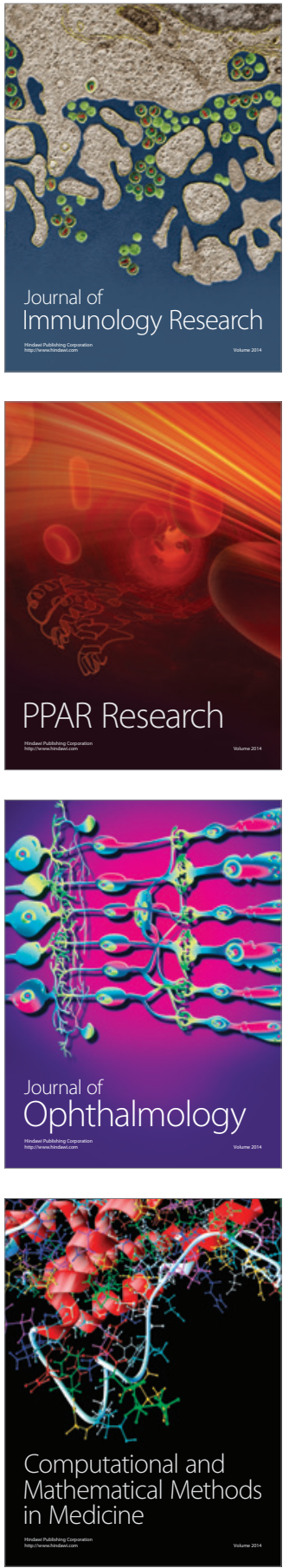

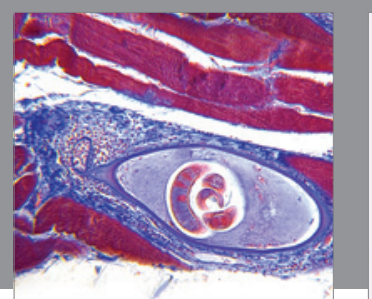

Gastroenterology Research and Practice

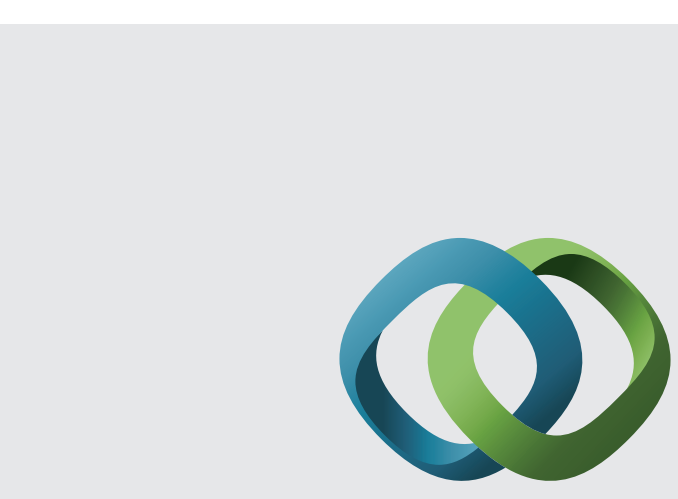

\section{Hindawi}

Submit your manuscripts at

http://www.hindawi.com
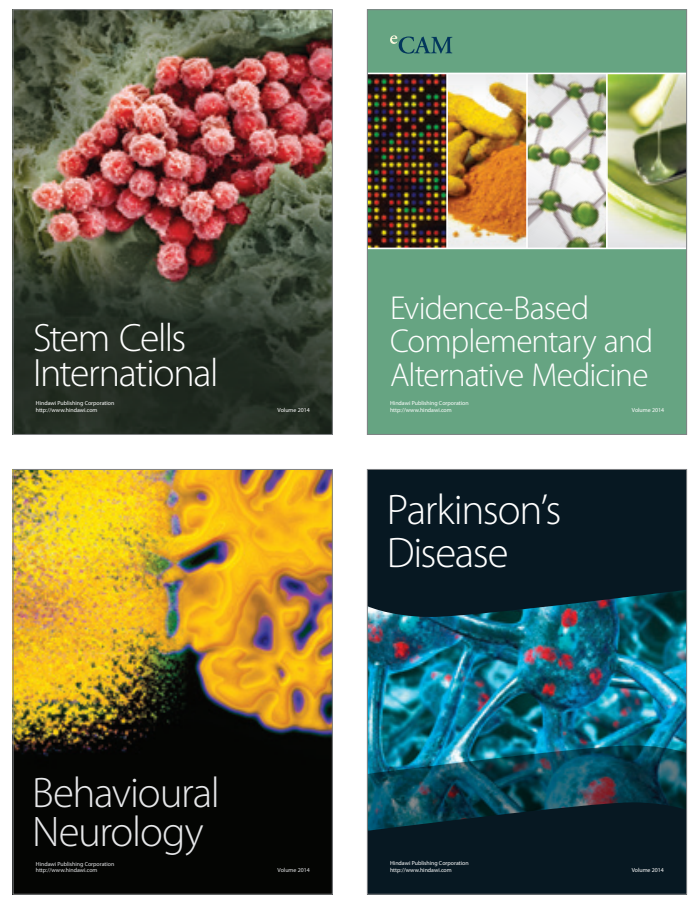
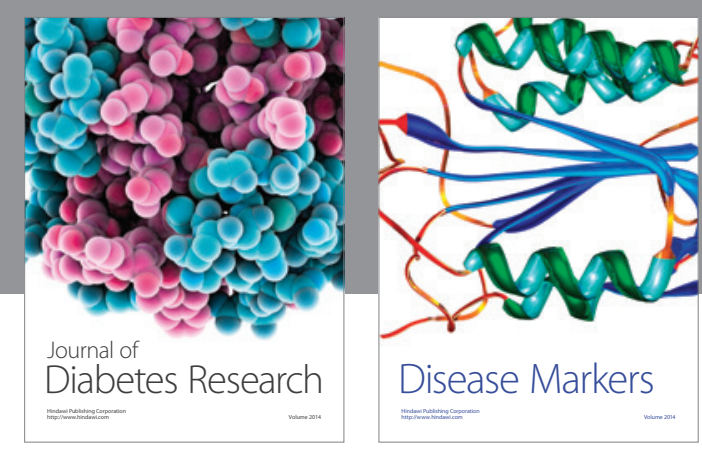

Disease Markers
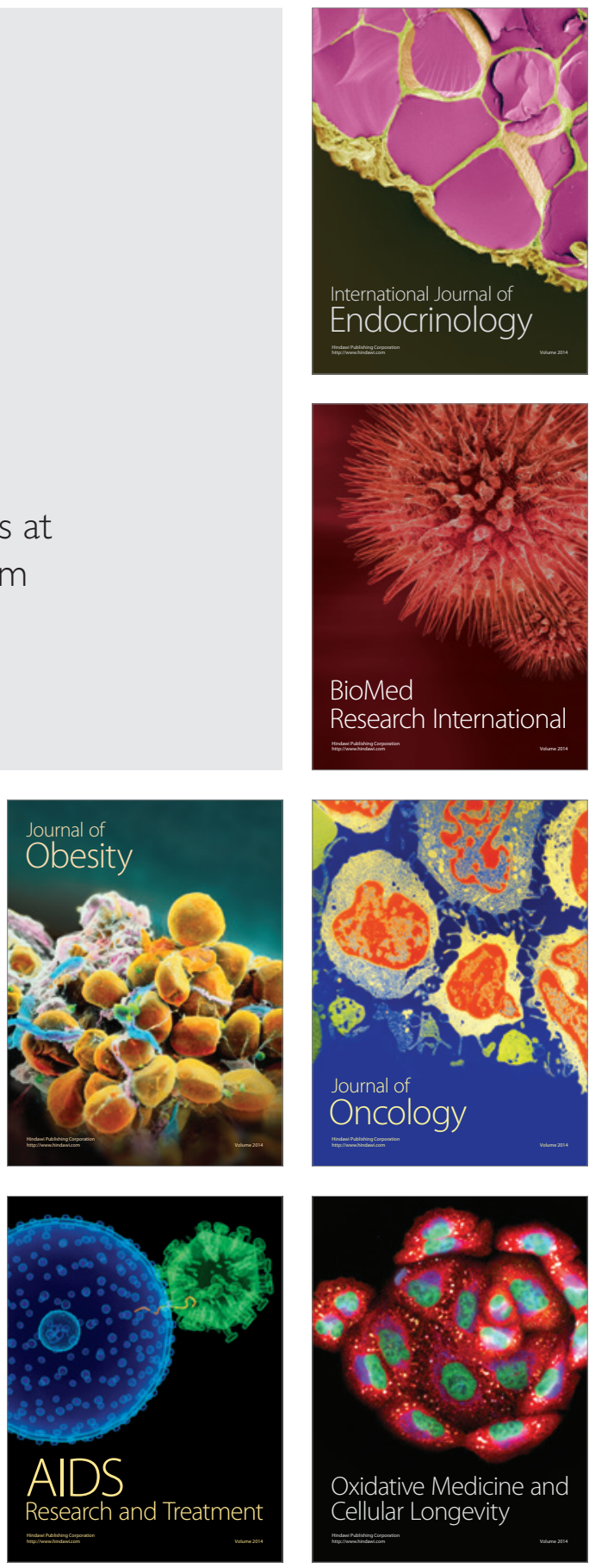